\title{
Una tribus ratio: Ikonographie der Wissensvermittlung und Selbstdarstellung der Jesuiten im Mathematiksaal des Kollegs Santo Antão in Lissabon
}

\author{
Samuel Gessner · Henrique Leitão
}

Eingegangen: 23. Dezember 2013 / Angenommen: 17. Januar 2014 /

Online publiziert: 21. Oktober 2014

(C) Springer-Verlag Berlin Heidelberg 2014

Das ehemalige Jesuitenkolleg Santo Antão in Lissabon verfügt über einen Mathematiksaal aus dem 18. Jahrhundert. Über seine Wände zieht sich an der unteren Seite rund herum ein Bilderzyklus aus Azulejos, der verschiedenartigste, auf die mathematischen Wissenschaften anspielende Motive darstellt (Abb. 1). ${ }^{1}$ Azulejos sind ein in Portugal viel verwendetes Mittel, Wandflächen zu verkleiden und zu verzieren. ${ }^{2}$ Außerdem gab es bei den Jesuiten europaweit eine Tradition der Bebilderung von Schulgebäuden und dabei insbesondere der allegorischen Darstellung verschiedener Lehrbereiche. ${ }^{3}$ So reichhaltige Zyklen mit mathematischen Themen, und besonders auf Azulejos, gibt es allerdings wohl nur in Portugal. ${ }^{4}$

Wozu wurde dieser Saal so aufwendig ausgestattet? Hängen die ausgewählten Themen mit den damals vor Ort vermittelten Lehrinhalten zusammen? Was bedeuten die dargestellten Szenen? Im Hinblick auf diese Fragen wird hier eines der Bilder genauer untersucht, bei dem vier Putti zu sehen sind, die mit einem Zylinder, einem Kegel und

S. Gessner $(\bowtie) \cdot$ H. Leitão

CIUHCT, Universidade de Lisboa, Lisbon, Portugal

E-Mail: samuel.gessner@gmail.com

\footnotetext{
${ }^{1}$ Rosário Salema de Carvalho, Samuel Gessner und Luís Tirapicos, „Astronomy and Azulejo panels in Portuguese Jesuit colleges“, in: F. Pimenta, N. Ribeiro, F. Silva, N. Campion, A. Joaquinito und L. Tirapicos (Hrg.), Stars and Stones. Proceedings of the SEAC 2011 Meeting, Oxford, Archaeopress, 2014, S. $284-287$. ${ }^{2}$ J. M. dos Santos Simões, Azulejaria em Portugal no Século XVIII, Lissabon, Fundação Calouste Gulbenkian, 1979. Weitere Hinweise und Literatur siehe Henrique Leitão \& Samuel Gessner, „Euclid in Tiles: The mathematical azulejos of the Jesuit college in Coimbra“, Math. Semesterberichte, 61 (2014) 1- 5.

${ }^{3}$ Solche thematische Ausschmückung von Lehrsälen entspricht einer Tradition in Anstalten der Jesuiten im Lauf des 17. und 18. Jahrhunderts. Barbara Bauer, „Experimentalphysik und Theologie. Die Embleme im mathematisch-physikalischen Museum zu Dillingen und die Physik P. Berthold Hausers SJ“, in: Scientia Poetica. Jahrbuch für Geschichte der Literatur und der Wissenschaften, Band. 5/2001, S. 35- 89.

${ }^{4}$ Ähnliche Motive auf Azulejos, auch zu anderen Wissenschaftszweigen, sind im Colégio do Espírito Santo in Évora, und weniger ausführlich im Palácio das Necessidades, im Palácio da Fronteira, und dem Convento de Jesus, der heutigen Academia das Ciências in Lissabon zu finden.
} 


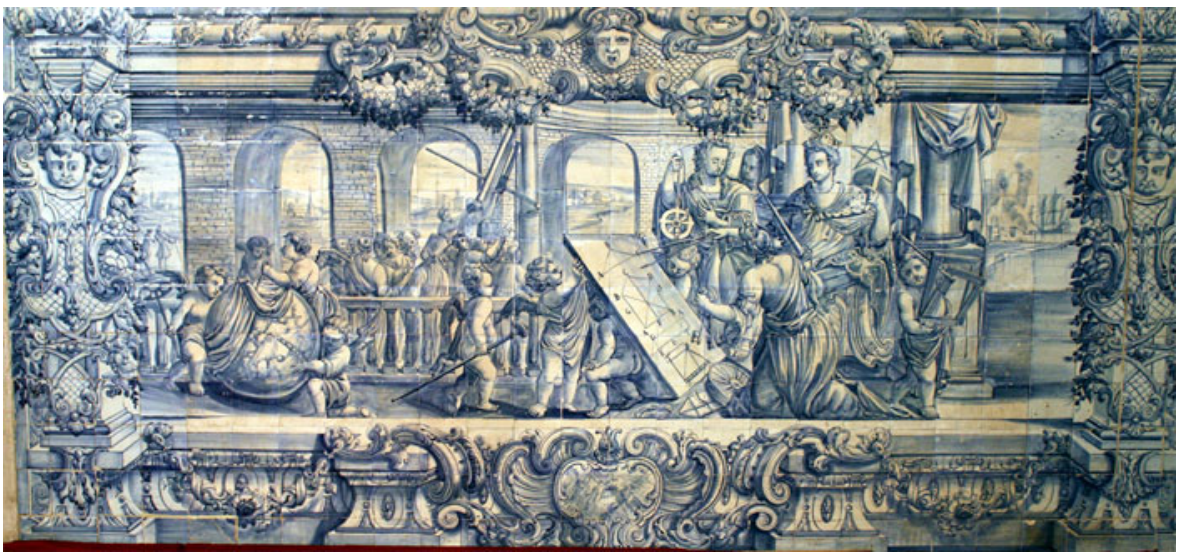

Abb. 1 Ein Hauptpaneel aus Azulejos, auf einem Kupferstich aus „Nouveaux élémens des mathématiques" von Prestet (1695) basierend, im Mathematiksaal des ehemaligen Jesuitenkollegs Santo Antão in Lissabon, heute Krankenhaus Hospital São José (Bilder der Azulejopaneele wurden freundlich überlassen durch das Projekt ,Az Infinitum - Azulejo. Indexation and Referencing System“. Siehe http://redeazulejo.fl.ul.pt/pesquisa-az)

einer Kugel spielerisch hantieren. Im Folgenden soll gezeigt werden, wie ein einzelnes, emblemartiges Motiv, dazu dienen konnte, nicht nur einen mathematischen Satz sondern auch klassische Bildung zu evozieren.

Der Mathematiksaal wurde über dem Eingang zur Kirche des Jesuitenkollegs eigens für die sogenannte Aula da Esfera (Halle der Sphärenlehre, Katheder für Sphaera) oder Academia Real de Matemática errichtet. Die Aula da Esfera war ohne Zweifel die bedeutsamste Einrichtung für die mathematische Ausbildung in der gesamten Geschichte Portugals, wobei die sogenannte „Praktische Mathematik“ im Vordergrund stand. ${ }^{5}$ Vorlesungen fanden, von den Anfängen der Institution (um 1592) bis zu ihrem Ende (1759) im Jesuitenkolleg in Lissabon (Colégio de Santo Antão) statt und wurde von Jesuitenpatern aus Portugal, Italien, Deutschland, Böhmen, England, Irland und den Niederlanden erteilt. Der Unterricht erfolgte auf Portugiesisch (und nicht auf Lateinisch), nämlich mit Rücksicht darauf, dass zu den Hörern nebst im Kolleg wohnenden Schülern auch externe Personen (Kartographen, Seefahrer, Instrumentenbauer, Architekten und andere Techniker) zählten.

Der entsprechende Teil des Kolleggebäudes hat sowohl das Erdbeben 1755 als auch die Verbannung der Jesuiten aus Portugal im Jahre 1759 überstanden, so dass die Aula da Esfera in ihrer letzten Ausstattung von ca. 1740 erhalten geblieben ist. Über ein barockes Treppenhaus ist der Saal von der Straße aus zu erreichen, so dass die

\footnotetext{
${ }^{5}$ Ugo Baldini, „L'insegnamento della matematica nel Collegio di S. Antão a Lisbona, 1590-1640“, in A companhia de Jesus e a missionação no Oriente, Akten des Kolloquiums, 21-23 April 1997, Braga, Barbosa \& Xavier Lda, 2000, S. 275-310; idem, „The teaching of mathematics in the Jesuit colleges of Portugal, from 1640 to Pombal", in L. Saraiva, H. Leitão (Hrg.), The practice of mathematics in Portugal, Coimbra, 2004; Henrique Leitão, A Ciência na Aula da Esfera do Colégio de Santo Antão, 1590-1759, Lissabon, Comissariado Geral das Comemorações do V Centenário do Nascimento de S. Francisco Xavier, 2007; Bernardo Mota, O estatuto da matemática em Portugal nos séculos XVI e XVIII, Lissabon, Fundação Calouste Gulbenkian, 2011, S. 203-312.
} 
Abb. 2 Nebenmotiv aus Azulejos im Mathematiksaal des Jesuitenkollegs in Lissabon

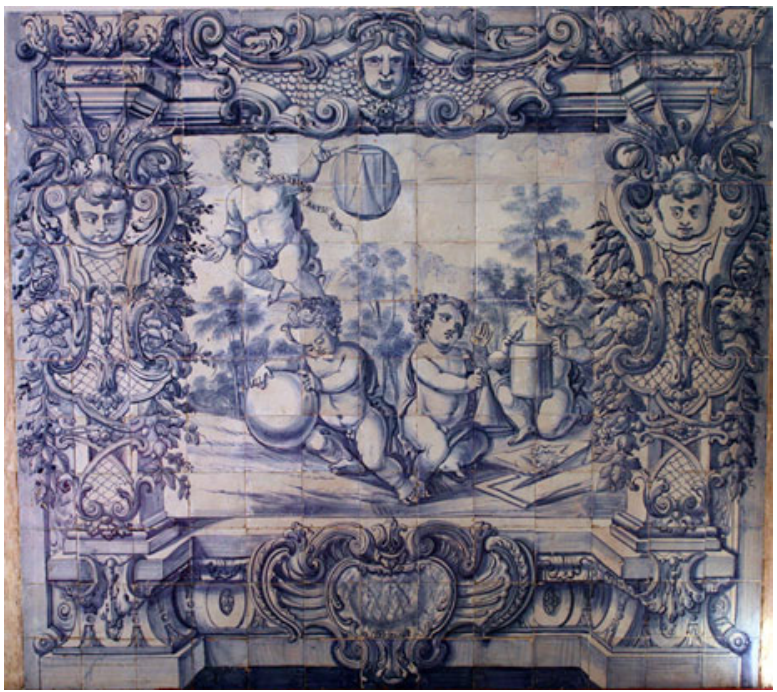

externen Studenten oder bei öffentlichen Anlässen, wie Disputationen oder Thesenverteidigungen, die Gäste leicht hineingelangen konnten, ohne den jungen Kollegiaten in die Quere zu kommen.

Die bildlichen Darstellungen in der Aula da Esfera umfassen Personifikationen der Festungsbaulehre, der Astronomie, der Nautik und der Geometrie, enthalten aber auch Illustrationen von Stadtbelagerungen sowie Allegorien zum weltweiten Wirken des Jesuitenordens. Hierbei sticht ins Auge, dass ,,archimedische“ Motive wiederholt auftreten: so etwa die Verteidigung von Syrakus mit Brennspiegeln, sodann ein Flaschenzug, mit dem die Welt aus den Angeln gehoben wird, und schließlich die Darstellung, die uns hier im Folgenden beschäftigt. Die Motive wurden nachweislich Kupferstichen in mathematischen Büchern entnommen, wobei vor allem die dritte Auflage der Nouveaux élémens des mathématiques (Paris 1695) des Priesters und Mathematikers Jean Prestet (ca. 1648-1690) und der Traité de la construction et des principaux usages des instrumens mathématiques (Paris 1709) von Nicolas Bion (ca. 1652-1733) zu nennen sind. ${ }^{6}$ Im weiteren Sinne haben alle Themen durchaus eine Beziehung zur praktischen Mathematik.

An einer der Seitenwände zwischen den Fenstern sind - in einer kleinen illusionistisch dargestellten barocken Umrahmung - vier Putten mit geometrischen Körpern dargestellt (Abb. 2). Drei von ihnen sitzen in einer baumgesäumten Lichtung und halten je einen Zylinder, einen Kegel und eine Kugel in Händen und hantieren mit Stechzirkeln. Vor ihnen liegt die Planskizze einer sternförmigen Festungsanlage, davor ein Winkeleisen und ein weiterer Zirkel. Über ihnen schwebt der vierte Putto, der auf die ebenfalls schwebenden drei geometrischen Körper hinweist, wobei sie nun ineinander verschachtelt erscheinen. Aus seinem Mund windet sich das Spruchband mit der Aufschrift: „Una tribus ratio est““ (Ein einziges Verhältnis [gilt] den Dreien).

\footnotetext{
${ }^{6}$ Salema, Gessner, Tirapicos, op. cit. siehe Anmerkung 1.
} 
Abb. 3 Titelblatt des Appendix mit archimedischen Lehrsätzen aus den „Elementa Geometriae“ (1654) des Jesuiten André Tacquet

\section{A N D R E 兔 \\ TA CQUET \\ E SOCIETATE IESU \\ SELECTA EX \\ A R C H I M E D E \\ THEOREMATA: \\ Via faciliori ac breviori demon- frata, or novis inventis aucta.

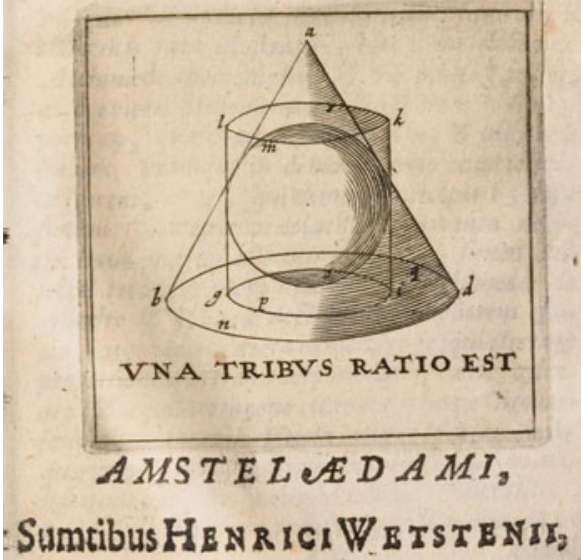

Die Darstellung und der Sinnspruch „Una tribus ratio est“ können auf den Satz der klassischen Stereometrie bezogen werden, wonach Inhalt und Oberfläche eines umschriebenen Zylinders zur eingeschriebenen Kugel im Verhältnis 3 zu 2 stehen. Außerdem bewies der Jesuit André Tacquet (1612-1660), der in Löwen und Antwerpen Mathematik unterrichtete, eine Erweiterung dieses archimedischen Satzes, dass nämlich das Volumen und die Oberfläche eines der selben Kugel umschriebenen „gleichseitigen“ Kegels zu jenem Zylinder wieder in demselben Verhältnis stehen. ${ }^{7}$ Tacquet setzt in seinen mehrfach aufgelegten und übersetzten Elementa geometriae planae ac solidae (Antwerpen 1654) mit diesem Theorem, Nr. 45, den Höhepunkt.

Die Verbindung der Azulejos zu Tacquets Lehrsatz ist insofern eindeutig, als das Epigramm „Una tribus ratio est“ in den Elementa auf dem Titelblatt des Appendix

\footnotetext{
${ }^{7}$ Der Öffnungswinkel des gleichseitigen Kegels beträgt $60^{\circ}$.
} 
Abb. 4 Ausschnitt aus dem oben gezeigten Azulejo-Bild

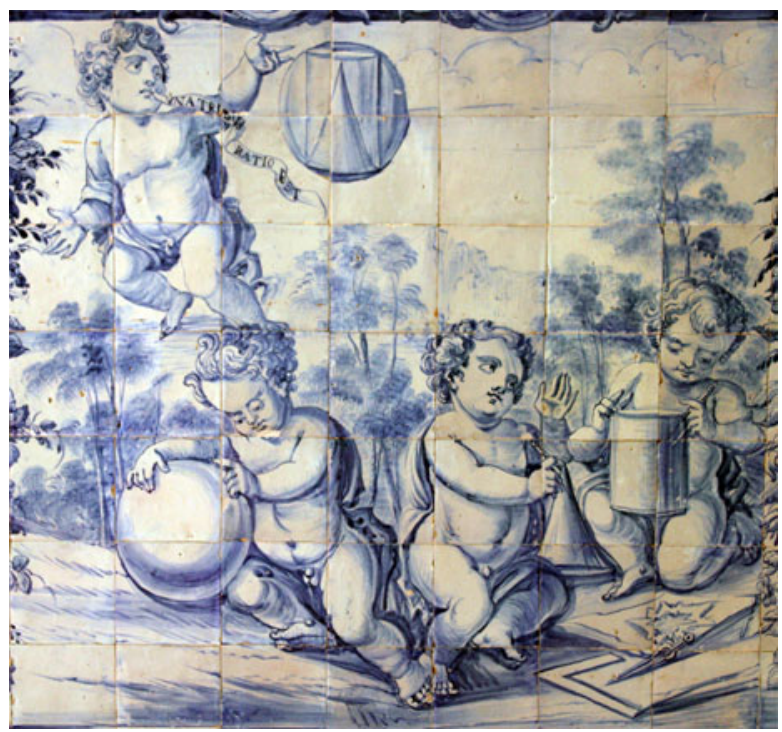

„Selecta ex Archimede theoremata“ zu lesen ist (Abb. 3), in dem der Autor einige archimedische Theoreme auf seine (angeblich einfachere und kürzere) Weise behandelte. Tacquets Elementa wurden in den Jesuitenkollegien in Portugal im Mathematikunterricht spätestens ab 1693 verwendet (oder dafür empfohlen), das belegen die Anweisungen des Jesuitengenerals zur Verbesserung des Mathematikunterrichts in der Portugiesischen Provinz. Daher erstaunt nicht, dass dieses Thema auch auf den Azulejos aufgegriffen wurde. Tacquet erklärt die Erweiterung seines Werks durch einige Theoreme von Archimedes damit, dass es sich bei Archimedes um den ruhmreichsten aller Mathematiker handle. Er verspricht seinen jungen Geometrieschülern, dass sie bei Archimedes erleben werden, wie viel ihnen Euklid nütze. ${ }^{8}$ Der Satz 45 , in seiner erstaunlichen Simplizität, mochte als Krönung der euklidischen geometrischen Grundlagen, und als Inbegriff ,,angewandter“ Theorie aufgefasst worden sein.

Das Nachwirken von Archimedes hat auch Tradition in der humanistischen Bildung. Auf Letztere dürfte die zur Schau gestellte Figur der drei Körper wohl auch noch auf einer zweiten Deutungsebene verweisen. Sowohl bei Cicero (Tusculanae disputationes 5.64) als auch bei Plutarch (Marcellus, Kap. 17) liest man von Archimedes' Wunsch, dass auf seinem Grabmal ein einer Kugel umschriebener Zylinder abgebildet werde, zusammen mit der Angabe des Verhältnisses, das sowohl für die Volumen als auch für die Oberflächen gilt. Das Motiv gibt also auch Gelegenheit, große Autoren der antiken Tradition zu zitieren. Damit wird auf kleinstem Raum die Einheit des jesuitischen Bildungsideals vorgeführt, das sowohl Mathematik wie auch Rhetorik und Philologie umfasste.

Wir vermuten, dass dasselbe Emblem noch eine dritte Deutungsebene zuließ, wonach das grammatische Subjekt des Epigramms vollkommen anders gesetzt worden

\footnotetext{
${ }^{8}$ Tacquet, „Lectori“, in Elementa geometriae planae ac solidae, Antwerpen, Jacobus Meursius, 1654, S. 279.
} 
wäre. ${ }^{9}$ Das Epigramm „Una tribus ratio est“ dürfte nämlich auch theologisch auslegbar gewesen sein und auf die Dreieinigkeit bezogen werden: Der Trias (Vater, Sohn und Heiliger Geist) ist ein und dasselbe Prinzip (ratio) gemeinsam.

Bei der Figur der ineinander verschachtelten Körper auf den blau-weißen Azulejos erkennt man nun aber bei genauerem Hinschauen, dass sie sich von der bei Tacquet unterscheidet: die Anordnung wurde invertiert! Der Zylinder ist hier einer Kugel eingeschrieben, und diesem wiederum eingeschrieben erscheint der Kegel (Abb. 4). Ein Fehler? Oder gilt wohl auch bei dieser Anordnung ein und dasselbe Verhältnis zwischen den Volumen und den Oberflächen? Was das Volumen angeht, so steht der Zylinder zum eingeschriebenen Kegel im Verhältnis 3 zu 1. Aber gibt es auch Zylinder, die so proportioniert sind, dass die ihnen umschriebene Kugel zum Zylinderinhalt 3 zu 1 steht? Das Problem hat zwei Lösungen: die eine, mit einem schlanken hohen Zylinder wie ihn die Azulejos abbilden; eine zweite mit einem plattgedrückten Zylinder. Allerdings stehen hier die Radien und Höhen in keinem einfachen rationalen Verhältnis. ${ }^{10}$ Ist diese Abweichung der Azulejos von Tacquets Vorlage absichtlich eingeführt worden oder konnte sie durch ein Missverständnis zwischen den Auftraggebern, den Entwurfskünstlern und den Fliesenmalern entstehen?

Wo immer auch die Ursache für die Abwandlung zu suchen ist, es ging damit die Schönheit und Einfachheit von Tacquets Lehrsatz verloren. Während bei Tacquet das Bild auf dem Titelblatt doch den Betrachter auf ein äußerst attraktives Resultat der Geometrie hinweist, bringen die Azulejos eine dem Auge vielleicht angenehmere, wenn auch komplexe geometrische Konfiguration zum Ausdruck. Die Figur hat aber nicht ihre echte Form, welche die eigentlich elegante und einfache ist. Wie so oft bei der Übertragung eines wissenschaftlichen Inhalts in eine für den allgemeinen Betrachter verständliche Form blieb hier der wahre Gehalt des Satzes auf der Strecke.

\footnotetext{
${ }^{9}$ Für diese Art Bedeutungsambivalenz der für Embleme typischen Auslegungspraxis siehe Bauer, op. cit., S. 42, Anmerkung 3.

${ }^{10}$ Die Halbhöhe h des eingeschriebenen Zylinders verhält sich zum Radius der umschriebenen Kugel wie $\mathrm{h}: 1$, wobei $\mathrm{h}^{3}-\mathrm{h}+\frac{2}{9}=0$. 